\title{
Influence of severity of infection on the effect of appropriate antimicrobial therapy for Acinetobacter baumannii bacteremic pneumonia
}

Fang-Yu Kang ${ }^{1}$, Chorng-Kuang How ${ }^{1,2}$, Yung-Chih Wang ${ }^{3}$, Aristine Cheng ${ }^{4}$, Ya-Sung Yang ${ }^{3}$, Shu-Chen Kuo ${ }^{5}$, Chang-Pan Liu ${ }^{6,7}$, Yuag-Meng Liü, Te-Li Chen ${ }^{9,10}$ and Yi-Tzu Lee ${ }^{1,2^{*}}$ (D)

\begin{abstract}
Background: The impact of appropriate antimicrobial therapy for A. baumannii bacteremic pneumonia has not been well established due to the inclusion of the three phenotypically indistinguishable Acinetobacter species and confounding factors including underlying diseases and severity of infection. This retrospective study aimed to evaluate the impact of appropriate antimicrobial therapy on 14-day mortality in A. baumannii bacteremic pneumonia patients after adjusting for risk factors.

Methods: This study was conducted at five medical centers in Taiwan between July 2012 and June 2016. A. baumannii species identification was performed using reference molecular methods. Risk factors for 14-day mortality were analyzed via logistic regression. The interaction between the Acute Physiology and Chronic Health Evaluation (APACHE) II score and appropriate antimicrobial therapy was assessed using the logistic model.

Results: A total of 336 patients with monomicrobial A. baumannii bacteremic pneumonia were included in this study. The overall 14-day mortality rate was $47.3 \%$. The crude mortality of appropriate antimicrobial therapy was 35.9\% (57 of 151 patients). Appropriate antimicrobial therapy was associated with a lower mortality after multivariate adjustment (odds ratio [OR], 0.57; 95\% confidence interval [CI], 0.34-0.97; $p=0.04$ ), and the effect was influenced by APACHE II score (OR for interaction term, $0.0098 ; 95 \% \mathrm{Cl}, 0.0005-0.1885 ; p=0.002$ ). Further analysis demonstrated that appropriate antimicrobial therapy significantly reduced 14-day mortality among the patients with an APACHE II score > 35 (OR 0.0098; 95\% Cl 0.0005-0.1885).
\end{abstract}

Conclusion: Appropriate antimicrobial therapy decreases 14-day mortality of the most severely ill patients with $A$. baumannii bacteremic pneumonia.

Keywords: Appropriate antimicrobial therapy, Acinetobacter baumannii, Bacteremia, Pneumonia, Severity

\footnotetext{
* Correspondence: s851009@yahoo.com.tw

'Department of Emergency Medicine, Taipei Veterans General Hospital, No.201, Section 2, Shipai Road, Taipei 11217, Taiwan

${ }^{2}$ Faculty of Medicine, School of Medicine, National Yang-Ming University, Taipei, Taiwan

Full list of author information is available at the end of the article
}

(C) The Author(s). 2020 Open Access This article is licensed under a Creative Commons Attribution 4.0 International License, which permits use, sharing, adaptation, distribution and reproduction in any medium or format, as long as you give appropriate credit to the original author(s) and the source, provide a link to the Creative Commons licence, and indicate if changes were made. The images or other third party material in this article are included in the article's Creative Commons licence, unless indicated otherwise in a credit line to the material. If material is not included in the article's Creative Commons licence and your intended use is not permitted by statutory regulation or exceeds the permitted use, you will need to obtain permission directly from the copyright holder. To view a copy of this licence, visit http://creativecommons.org/licenses/by/4.0/. The Creative Commons Public Domain Dedication waiver (http://creativecommons.org/publicdomain/zero/1.0/) applies to the data made available in this article, unless otherwise stated in a credit line to the data. 


\section{Background}

Nosocomial pneumonia is one of the main causes of mortality and morbidity among hospitalized patients [1, $2]$. The estimated attributable mortality ranges between 33 and 50\% [3]. Acinetobacter baumannii is one of the leading pathogens of nosocomial pneumonia worldwide and is associated with poorer outcomes [4-7]. Although it is difficult to determine the attributable mortality due to severe comorbidities $[5,8]$, many studies have shown that the high levels of resistance of $A$. baumannii to antimicrobials may play an important role [8-12]. The high antimicrobial resistance of $A$. baumannii leads to higher rates of inappropriate empirical antimicrobial therapies, and may contribute to a greater risk of death $[13,14]$.

Numerous studies have investigated the efficacy of antimicrobial therapy for A. baumannii bacteremia [1518]. Appropriate antimicrobial therapy is associated with a lower mortality rate in A. baumannii bacteremia patients $[15,17]$, and the therapeutic effects might be more significant in severely ill patients [18]. The impact of appropriate antimicrobial therapy for $A$. baumannii bacteremic pneumonia has not been well established. Many factors contribute to the mortality of A. baumannii bacteremic pneumonia, and it remains unclear whether appropriate antimicrobial therapy increases survival among all patients or only among patients with certain demographic or clinical characteristics. Furthermore, the association between appropriate antimicrobial therapy and mortality for A. baumannii bacteremic pneumonia has been difficult to establish due to the confounding influence of the three phenotypically indistinguishable Acinetobacter species that make up the A. baumannii (Ab) group (A. baumannii, Acinetobacter nosocomialis and Acinetobacter pittii). The aims of this retrospective study were to evaluate the impact of appropriate antimicrobial therapy on the 14-day mortality in genomically identified $A$. baumannii bacteremic pneumonia patients, and to determine if the therapeutic effect of appropriate antimicrobial therapy differed between patients with different infection severities.

\section{Materials and methods}

\section{Data collection and patients}

This study was conducted at the following five medical centers in Taiwan: Taipei Veterans General Hospital (TVGH, 2900 beds), Tri-Service General Hospital (TSGH, 1712 beds), Mackay Memorial Hospital (MMH, 2055 beds), and National Taiwan University Hospital (NTUH, 2200 beds) in Northern Taiwan and Changhua Christian Hospital (CCH, 1676 beds) in Central Taiwan. Data was collected between July 2012 and June 2016. The inclusion criteria for $A$. baumannii bacteremic pneumonia were: (1) $\geq 1$ positive blood culture for $A$. baumannii which could not be attributed to an infection source other than the lower respiratory tract; (2) a clinical course compatible with the diagnosis of pneumonia, including a new pulmonary infiltrate plus one additional criterion (fever $\geq 38{ }^{\circ} \mathrm{C}$, blood leukocytosis $\geq 10,000$ cells/ $\mathrm{mm}^{3}$ or leucopenia $\leq 3000$ cells $/ \mathrm{mm}^{3}$ ), together with one or more of the following conditions: new cough, change in sputum color, chest pain, and dyspnea; (3) $\geq 1$ positive respiratory sample (sputum, endotracheal aspirate, or broncho-alveolar lavage [BAL]) for A. baumannii collected within $48 \mathrm{~h}$ before or after the first positive blood culture for A. baumannii. Patients below 20 years of age or without a complete medical record were excluded. The study protocol was approved by the Research Ethics Committee of all participating hospitals. Informed consent was waived because of the retrospective nature of the study and the analysis used anonymous clinical data.

\section{Study variables and definitions}

The following data were collected from patient's medical records: demographic information, comorbid conditions, duration of hospital and intensive care unit (ICU) stays, time, dose and route of antimicrobial therapy, the use of ventilator, and procedures (central venous catheters, arterial catheters, foley catheter, nasogastric tube, hemodialysis, and tracheostomy) at the time of bacteremia.

The onset of bacteremia was defined as the day the positive blood culture of A.baumannii was collected. The bacteremic pneumonia was considered acquired in the ICU if the positive respiratory sample for A.baumannii and positive blood culture for A.baumannii were both obtained at least $48 \mathrm{~h}$ after ICU admission. Previous ICU admission was defined as being admitted to ICU within 4 weeks prior to the onset of bacteremia.

Immunosuppressive therapy was defined as receiving cytotoxic agents within 6 weeks, corticosteroids at a dosage equivalent to or higher than $15 \mathrm{mg}$ of prednisolone daily for 1 week within 4 weeks, or other immunosuppressive agents within 2 weeks of the onset of bacteremia. Chronic kidney disease was defined as an estimate glomerular filtration rate $<60 \mathrm{~mL} / \mathrm{min} / 1.73 \mathrm{~m}^{2}$. Neutropenia referred to an absolute neutrophil count < $0.5 \times 10^{9}$ neutrophils/L. Recent surgery was defined as undergoing an operation within 4 weeks of the onset of bacteremia. Previous ventilator use was defined as mechanical ventilation use for more than 3 days in the past 4 weeks. The severity of patient illness was evaluated using the Acute Physiology and Chronic Health Evaluation (APACHE) II score within $24 \mathrm{~h}$ before bacteremia onset.

Appropriate antimicrobial therapy was defined as administration of the antimicrobial agent to the pathogen susceptible in vitro, within $48 \mathrm{~h}$ after the onset of bacteremia, with an approved route and dosage 
appropriate for end organ(s) function. Antimicrobial therapy that did not meet this definition was considered inappropriate. Monotherapy with an aminoglycoside was also considered to be an inappropriate therapy. An antimicrobial agent (or antimicrobial agents)-based therapy was defined as treatment with the antimicrobial agent(s) alone or in combination with other antimicrobial agent(s). The colistin loading dose was $5 \mathrm{mg} / \mathrm{kg}$ colistin base activity, followed by $5 \mathrm{mg} / \mathrm{kg} / \mathrm{d}$ colistin base activity divided over 8 or $12 \mathrm{~h}$ in patients with normal renal function. For those with impaired renal function, the dosage was adjusted according to renal function as previously described $[19,20]$. The loading dose of tigecycline was $100 \mathrm{mg}$, followed by a maintenance dose of 50 mg every $12 \mathrm{~h}$. The primary outcome was all-cause 14day mortality after the onset of $A$. baumannii bacteremia.

\section{Microbiological studies}

The presumptive identification of the isolates to the level of the $A$. baumannii complex was determined using the Vitek 2 system (bioMérieux). All $A$. baumannii complex bloodstream isolates were regrown from storage, identified to species level, and tested for their susceptibility to various antimicrobials. A multiplex polymerase chain reaction method was used to identify $A$. baumannii to the genomic species level [21]. Polymicrobial bacteremia was defined as the concurrent isolation of one or more microorganisms other than A. baumannii from blood. Antimicrobial susceptibility to ampicillin-sulbactam, ceftazidime, cefepime, piperacillin-tazobactam, imipenem, meropenem, ciprofloxacin, levofloxacin, and amikacin was determined by the agar dilution method according to Clinical Laboratory Standards Institute criteria. Colistin minimal inhibitory concentrations (MICs) were determined by the broth macrodilution method to problems arising from the fact that the surface charge on the polystyrene microplate applied during manufacturing influences the level of colistin adsorption to the plate surface [22, 23] Tigecycline MICs were determined by the broth microdilution method using fresh medium [24]. Multidrug resistance (MDR) was defined as resistance to at least one agent in at least three of the following classes of antimicrobials: antipseudomonal cephalosporins, antipseudomonal carbapenems, ampicillin-sulbactam, fluoroquinolones, and aminoglycosides. Carbapenem resistance was defined as resistance to imipenem or meropenem. Extensive drug resistance (XDR) referred to non-susceptibility to imipenem or meropenem and all drug classes with the exception of colistin and tigecycline.

\section{Statistical analysis}

Chi-squared test or Fisher's exact test was used to compare categorical variables. The Student's t test or ManWhitney rank sum test was used to analyze continuous variables. Logistic regression models were used to assess independent risk factors for 14-day mortality. Biologically plausible variables which were significantly associated with mortality $(p \leq 0.05)$ in the univariable analysis were included in the multivariable analysis. Stepwise logistic regression was used. Interactions between the APACHE II score and the covariates were assessed in the logistic regression model. APACHE II scores were categorized into four groups (APACHE II score $\leq 15$, > 15 and $\leq 25,>25$ and $\leq 35,>35$ ) in the logistic regression models based on their quartile distribution and previous studies $[18,25]$. The time between bacteremia onset to mortality was analyzed using Kaplan-Meier survival analysis. A $p$-value $<0.05$ was considered to be statistically significant. All the analyses were processed with Stata software version 12 .

\section{Results}

During the study period, 875 patients were found to have had at least one episode of bacteremia caused by $A$. baumannii. We excluded 164 patients with polymicrobial bacteremia and 375 patients with a positive blood culture attributable to another source of infection. A total of 336 patients who met the criteria of A. baumannii monomicrobial bacteremic pneumonia were included during the 4-year study period.

The overall 14-day mortality rate of A. baumannii bacteremic pneumonia was $47.3 \%$ (159 of 336 patients). The crude mortality of appropriate antimicrobial therapy was $35.9 \%$ (57 of 151 patients). The demographic and clinical characteristics are demonstrated in Table 1. The 14-day non-survivors were more likely to have hematological malignancies and have underwent immunosuppressive therapy but less likely to have cerebrovascular accident or recent surgery. Non-survivors had a significantly higher APACHE II score and higher rates of previous ventilator use. There was no significant difference in the rates of invasive procedures between the 14day survivor and non-survivors.

The bloodstream isolates of non-survivors had higher MDR, XDR and carbapenem resistance rates than those of survivors $(p<0.05)$. Survivors were significantly more likely to have received appropriate antimicrobial therapy than non-survivors. Factors that significantly predicted 14-day mortality in logistic regression are shown in Table 2. Multivariable analysis showed that administration of appropriate antimicrobial therapy was independently associated with lower mortality (OR, 0.57; $95 \% \mathrm{CI}$, 0.34-0.97; $p=0.04$ ). APACHE II score and XDR were independent predictors of 14-day mortality (both $p<$ 0.001).

Further exploration of the potential effect modification on the impact of appropriate antimicrobial therapy on 14-day mortality suggested that the severity of infection 
Table 1 Demographic and clinical characteristics of patients with Acinetobacter baumannii bacteremic pneumonia stratified by 14day mortality

\begin{tabular}{|c|c|c|c|}
\hline Characteristics & Survivors $(n=177)$ & Non-survivors $(n=159)$ & $P$-value \\
\hline \multicolumn{4}{|l|}{ Demographics } \\
\hline Male, No. (\%) & $128(72.3)$ & $122(76.7)$ & .36 \\
\hline Age, median (IQR), years & $70(67-72)$ & $69(66-72)$ & .64 \\
\hline Acquired in ICU, No. (\%) & $91(51.4)$ & $90(56.6)$ & .34 \\
\hline Previous ICU admission & $127(71.8)$ & $110(69.2)$ & .61 \\
\hline Length of hospitalization before bacteremia, median (IQR), days & $36(24-48)$ & $35(27-44)$ & .98 \\
\hline \multicolumn{4}{|l|}{ Comorbidities, No. (\%) } \\
\hline Charlson co-morbidity score & $3.8(3.4-4.2)$ & $3.9(3.5-4.2)$ & .75 \\
\hline Malignancy & $50(28.3)$ & $44(27.7)$ & .91 \\
\hline Solid tumor & $42(23.7)$ & $30(18.9)$ & .28 \\
\hline Hematologic malignancy & $8(4.5)$ & $21(13.2)$ & .005 \\
\hline Type 2 diabetes mellitus & $63(35.6)$ & $44(27.7)$ & .12 \\
\hline Cerebrovascular accident & $47(26.6)$ & $21(13.2)$ & .002 \\
\hline Hypertension & $85(48.0)$ & $65(40.9)$ & .19 \\
\hline Immunosuppressant use & $35(19.8)$ & $56(35.2)$ & .001 \\
\hline Liver cirrhosis & $19(10.7)$ & $14(8.8)$ & .55 \\
\hline Chronic kidney disease & $58(32.8)$ & $62(39.0)$ & .23 \\
\hline Coronary artery disease & $29(16.4)$ & $24(15.1)$ & .75 \\
\hline Congestive heart failure & $34(19.2)$ & $34(21.4)$ & 62 \\
\hline Chronic obstructive pulmonary disease & $39(22.0)$ & $33(20.8)$ & .78 \\
\hline Collagen vascular disease & $10(5.7)$ & $18(11.3)$ & .06 \\
\hline Chemotherapy & $13(7.3)$ & $21(13.2)$ & .08 \\
\hline Neutropenia & $8(4.5)$ & $13(8.2)$ & .17 \\
\hline Recent surgery & $64(36.2)$ & $32(20.1)$ & .001 \\
\hline \multicolumn{4}{|l|}{ Invasive procedures, No. $(\%)^{a}$} \\
\hline Arterial line & $63(35.6)$ & $69(43.4)$ & .14 \\
\hline Central venous catheter & $111(62.7)$ & $113(71.1)$ & .11 \\
\hline Hemodialysis & $21(11.9)$ & $26(16.4)$ & 024 \\
\hline Tracheostomy & $44(24.9)$ & $50(31.5)$ & .18 \\
\hline Ventilator (previous use) & $73(41.2)$ & $92(57.9)$ & .002 \\
\hline Ventilator (current use) & $121(68.4)$ & $121(76.1)$ & .12 \\
\hline Ventilator associated pneumonia & $121(68.4)$ & $113(71.1)$ & .59 \\
\hline \multicolumn{4}{|l|}{ Clinical condition } \\
\hline APACHE II score within $24 \mathrm{~h}$ before bacteremia, median (IQR) & $23(21-24)$ & $33(32-35)$ & $<.001$ \\
\hline Shock & $75(42.4)$ & $75(47.2)$ & .38 \\
\hline \multicolumn{4}{|l|}{ Resistance profiles of bloodstream isolates, No. (\%) } \\
\hline Multidrug resistance $(\mathrm{MDR})^{\mathrm{b}}$ & $156(88.1)$ & $150(94.3)$ & .046 \\
\hline Carbapenem resistance & $104(58.8)$ & $129(81.1)$ & $<.001$ \\
\hline Extensive drug resistance $(X D R)^{c}$ & $58(32.8)$ & $95(59.8)$ & $<.001$ \\
\hline Appropriate antimicrobial therapy & $94(53.1)$ & $57(35.9)$ & .001 \\
\hline
\end{tabular}

Abbreviations: APACHE II Acute Physiology and Chronic Health Evaluation II, ICU intensive care unit, IQR interquartile range

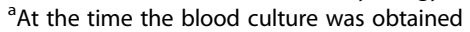

${ }^{b}$ Resistance to at least one agent in at least three of the following classes of antimicrobials: antipseudomonal cephalosporins, antipseudomonal carbapenems, ampicillin-sulbactam, fluoroquinolones, and aminoglycosides

${ }^{\mathrm{C}}$ Extensive drug resistance (XDR) referred to non-susceptibility to imipenem or meropenem and all drug class except for colistin and tigecycline 
Table 2 Logistic regression of predictors for 14-day mortality in patients with Acinetobacter baumannii bacteremic pneumonia

\begin{tabular}{|c|c|c|c|c|}
\hline \multirow[b]{2}{*}{ Characteristic } & \multicolumn{2}{|c|}{ Univariable Analysis } & \multicolumn{2}{|c|}{ Multivariable Analysis } \\
\hline & OR $(95 \% \mathrm{Cl})$ & $P$-value & OR $(95 \% \mathrm{Cl})$ & $P$-value \\
\hline Hematologic malignancy & $3.21(1.38-7.48)$ & .004 & & \\
\hline Cerebrovascular accident & $0.42(0.24-0.74)$ & .003 & $0.40(0.20-0.81)$ & .011 \\
\hline Immunosuppressant use & $2.21(1.35-3.61)$ & .002 & $1.73(0.95-3.16)$ & .072 \\
\hline Recent surgery & $0.44(0.27-0.73)$ & .001 & $0.50(0.27-0.92)$ & .025 \\
\hline Carbapenem resistance & $3.02(1.84-4.96)$ & $<.001$ & & \\
\hline Extensive drug resistance & $3.05(1.95-4.76)$ & $<.001$ & $3.19(1.86-5.46)$ & $<.001$ \\
\hline Previous ventilator use & $1.96(1.27-3.02)$ & .002 & & \\
\hline APACHE II score (categorical) & $3.47(2.57-4.68)$ & $<.001$ & $3.20(2.33-4.39)$ & $<.001$ \\
\hline Appropriate antimicrobial therapy & $0.49(0.32-0.76)$ & .001 & $0.57(0.34-0.97)$ & .04 \\
\hline
\end{tabular}

All biologically plausible variables with a $p$-value $<0.05$ in the univariable analysis were considered for inclusion in the logistic regression model in the multivariable analysis. A stepwise selection process was utilized. We found that only cerebrovascular accident, recent surgery, extensive drug resistance, APACHE II score, and appropriate therapy were statistically significant factors for 14-day mortality

Abbreviations: APACHE II Acute Physiology and Chronic Health Evaluation II, Cl confidence interval

is an effect modifier. Interactions between the APACHE II score and appropriate antimicrobial therapy were added to the logistic regression model. The interaction term was statistically significant (OR for interaction term, 0.0098; 95\% CI, 0.0005-0.1885; $p=0.001$ ). Table 3 demonstrates the adjusted ORs for appropriate antimicrobial therapy administered to four different severities of infection by APACHE II score categories. Appropriate antimicrobial therapy was not associated with a lower mortality among patients with APACHE scores $\leq 15$ or $>15$ and $\leq 25$ or $>25$ and $\leq 35$ (Groups I, II, and III). On the other hand, among those with APAC HE II scores $>35$ (Group IV), appropriate antimicrobial therapy significantly reduced the 14-day mortality (OR 0.0098; 95\% CI, 0.0005-0.1885). A similar magnitude of association and trend was also obtained when the APAC HE II score was categorized into four groups based on its quartile distribution (Supplemental Table S1). Among patients with APACHE scores $\leq 35$ (Groups I + II + III), appropriate antimicrobial therapy was not associated with a lower mortality by univariate and multivariate analysis (Supplemental Table S2). Subgroup analyses showed that among the patients who were admitted in the ICU at the time of bacteremia (101 patients), appropriate antimicrobial therapy lowers 14-day mortality in the patients with APACHE score > 35 (OR 0.023; 95\% CI 0.0015-0.3508). Of the patients who were ventilator assisted at the time of bacteremia (242 patients), those with an APACHE score $>35$ had a lower 14-day mortality rate if receiving appropriate antimicrobial therapy (OR 0.014; 05\% CI 0.0007-0.2812).

Kaplan-Meier survival curves were used to compare the impacts of receiving appropriate or inappropriate antimicrobial therapy on mortality, stratified by APAC HE II score groups as mentioned in Table 3. Although no significant differences in survival were noted between patients receiving appropriate versus inappropriate antimicrobial therapy in group I $(p=0.7106$, by log-rank test), II ( $p=0.9843$, by log-rank test) (figures not shown), and III ( $p=0.2014$, by log-rank test) (Fig. 1a), there was a significant advantage in survival for appropriate compared to inappropriate use of antimicrobial therapy in group IV ( $p<0.001$, by log-rank test) (Fig. 1b).

The effect of appropriate antimicrobial therapy on 28day survival was analyzed as per the above analyses. The results were similar to those found when using all-cause 14-day mortality as the primary outcome measure (data not shown).

The multivariate analysis of the demographic and clinical characteristics between patients receiving

Table 3 Adjusted odds ratios for appropriate antibiotics for 14-day mortality in patients with Acinetobacter baumannii bacteremic pneumonia: Stratified by APACHE II score categories

\begin{tabular}{llllll}
\hline Group & APACHE II score & Patients, No. & $\begin{array}{l}\text { 14-Day } \\
\text { Mortality (\%) }\end{array}$ & Adjusted OR ${ }^{\mathbf{a}} \mathbf{( 9 5 \% ~ C l )}$ & $\boldsymbol{P}_{\text {-value }}$ \\
\hline I & $<=15$ & 43 & 16.3 & $2.42(0.38-15.18)$ & 0.345 \\
II & $16-25$ & 108 & 26.9 & $0.83(0.33-2.13)$ & 0.704 \\
III & $26-35$ & 110 & 51.8 & $0.61(0.26-1.40)$ & 0.241 \\
IV & $>=36$ & 75 & 88.0 & $0.0098(0.0005-0.1885)$ & 0.002 \\
\hline
\end{tabular}

Abbreviations: APACHE II Acute Physiology and Chronic Health Evaluation II, Cl confidence interval, OR odds ratio

${ }^{a}$ Adjusted for cerebrovascular accident, immunosuppressant use, recent surgery, extensive drug resistance, APACHE II score, and appropriate therapy 

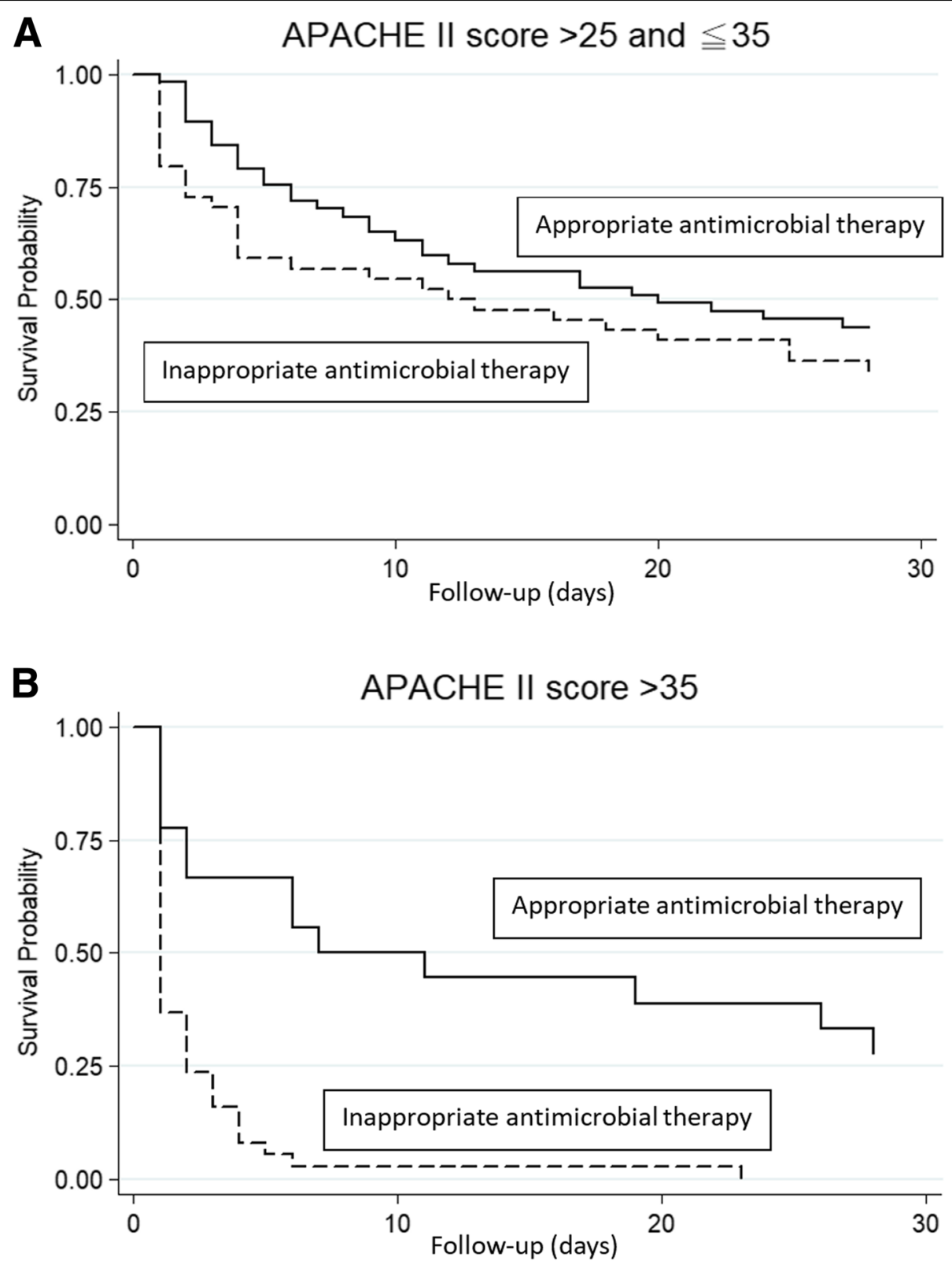

Fig. 1 Kaplan-Meier survival curves at 28 days after Acinetobacter baumannii bacteremic pneumonia onset for patient receiving appropriate or inappropriate antimicrobial therapy, stratified by severity of infection. A, Group III, with Acute Physiology and Chronic Health Evaluation II scores $>25$ and $\leq 35$. B, Group IV, with APACHE II score >35. Abbreviation: APACHE II, Acute Physiology and Chronic Health Evaluation II

appropriate (151 patients) or inappropriate (185 patients) antimicrobial therapy showed that those with a history of myocardial infarction $(p=0.045)$, higher APACHE II scores $(p=0.001)$, and extensive drug resistance $(p=0.029)$ were more likely to receive inappropriate antimicrobial therapy. On the other hand, patients who has history of connective tissue disease $(p=0.035)$ and received a central venous catheter at the time of bacteremia onset $(p=0.004)$ had a higher chance of receiving appropriate antibiotics.
The independent impact of appropriate antimicrobial therapy on 14-day mortality were further explored by including age, gender, extensive drug resistance, APACHE II score and all the comorbidities in Table 1 in the multivariable logistic regression model (Supplemental Table S3). The results were similar to those including the statistically significant predictors using stepwise logistic regression (Table 2). Further analysis showed that the interaction between APACHE II score and appropriate antimicrobial 
Table 4 Antimicrobial regimens for the treatment of Acinetobacter baumannii bacteremic pneumonia (appropriate antibiotics)

\begin{tabular}{|c|c|c|c|c|c|c|c|}
\hline \multirow[t]{2}{*}{ Main agents used ${ }^{a, b}$} & \multirow{2}{*}{$\begin{array}{l}\begin{array}{l}\text { No. }(\%) \\
\text { of } \\
\text { patients } \\
(n= \\
151)\end{array} \\
\end{array}$} & \multirow{2}{*}{$\begin{array}{l}\text { APACHE II } \\
\text { score, } \\
\text { median } \\
\text { (IQR) }^{d}\end{array}$} & \multicolumn{5}{|l|}{ No. (\%) of patients } \\
\hline & & & Combination therapy ${ }^{\mathrm{e}}$ & $\begin{array}{l}\text { 14-Day } \\
\text { Mortality }\end{array}$ & $P$-value & $\begin{array}{l}\text { 28-Day } \\
\text { Mortality }\end{array}$ & $P$-value \\
\hline Anti-pseudomonas penicillin-based therapy & $13(8.6)$ & $245(19-29)$ & $8(61.5)$ & $6(46.2)$ & 0.513 & $8(61.5)$ & .319 \\
\hline Anti-pseudomonas cephalosporin-based therapy & $31(20.5)$ & $25(19-32)$ & $24(77.4)$ & $10(32.3)$ & 0.479 & $12(38.7)$ & .229 \\
\hline Carbapenem-based therapy & $59(39.1)$ & $25(18-28)$ & $25(42.4)$ & $20(33.9)$ & 0.434 & $28(47.5)$ & .861 \\
\hline Colistin-based therapy & $55(36.4)$ & $25(19-29)$ & $49(89.1)$ & $28(50.9)$ & 0.012 & $33(60.0)$ & .030 \\
\hline Tigecycline-based therapy & $54(35.8)$ & $28(22-32)$ & $46(85.2)$ & $28(51.9)$ & 0.008 & $32(59.3)$ & .045 \\
\hline Fluoroquinolone-based therapy & $7(4.6)$ & $25(12-36)$ & $3(42.9)$ & $4(57.1)$ & 0.278 & $4(57.1)$ & .633 \\
\hline Sulbactam-based therapy & $24(15.9)$ & $26(22-28)$ & $13(54.2)$ & $7(29.2)$ & 0.344 & $10(41.7)$ & .475 \\
\hline Carbapenem + colistin-based therapy & $15(9.9)$ & $24(18-28)$ & $6(40.0)$ & $9(60.0)$ & 0.061 & $11(73.3)$ & .041 \\
\hline Carbapenem + tigecycline-based therapy & $12(8.0)$ & $25(22-28)$ & $7(58.3)$ & $9(75.0)$ & 0.006 & $10(83.3)$ & .011 \\
\hline Carbapenem + sulbactam-based therapy & $6(4.0)$ & $25(22-28)$ & $5(83.3)$ & $4(66.7)$ & 0.200 & $5(83.3)$ & .107 \\
\hline Colistin + tigecycline-based therapy & $25(16.6)$ & $27(20-32)$ & $12(48.0)$ & $14(56.0)$ & 0.039 & $15(60.0)$ & .202 \\
\hline Carbapenem + colistin + tigecycline-based therapy & $4(2.7)$ & $24(20-28)$ & $2(50.0)$ & $4(100.0)$ & 0.019 & $4(100.0)$ & .052 \\
\hline \multicolumn{8}{|l|}{ Antimicrobial regimens ${ }^{c}$} \\
\hline Anti-pseudomonas penicillin only & $5(3.3)$ & $24(19-30)$ & & $2(40.0)$ & 1.000 & $4(80.0)$ & .198 \\
\hline Anti-pseudomonas cephalosporin only & $7(4.6)$ & $23(16-30)$ & & $2(28.6)$ & 0.711 & $2(28.6)$ & .444 \\
\hline Carbapenem + colistin & $9(6.0)$ & $24(18-26)$ & & $4(44.4)$ & 0.730 & $6(66.7)$ & .315 \\
\hline Carbapenem + tigecycline & $5(3.3)$ & $25(26-28)$ & & $3(60.0)$ & 0.366 & $3(60.0)$ & .673 \\
\hline Carbapenem + sulbactam & $1(0.7)$ & 17 & & 0 & 1.000 & 0 & 1.000 \\
\hline Carbapenem + tigecycline + colistin & $2(1.3)$ & 17,22 & & $2(100)$ & 0.141 & $2(100)$ & .232 \\
\hline Tigecycline only & $8(5.3)$ & $29(28-33)$ & & $5(62.5)$ & 0.155 & $5(62.5)$ & .484 \\
\hline Colistin + tigecycline & $13(8.6)$ & $29(22-38)$ & & $7(53.9)$ & 0.210 & 7 (53.9) & .678 \\
\hline
\end{tabular}

${ }^{a} \mathrm{An}$ antimicrobial agent (or antimicrobial agents)-based therapy denotes the corresponding antimicrobial agent(s) alone or in combination with other antimicrobial agent(s)

b"Colistin" denotes intravenous colistin only. Inhaled colistin is not included

${ }^{\mathrm{C}}$ Not in combination with other antimicrobial agents

dIQR, interquartile range. When the case number is less than 4, the APACHE II score for each case is shown

${ }^{\mathrm{e}} \mathrm{C}$ (ombination therapy is defined as administration of more than one antimicrobial agent

therapy was statistically significant after adjusting for the demographic characteristics and comorbidities (Supplemental Table S4). The results were similar to the analysis using the statistically significant predictors in the regression model (Table 3), demonstrating that appropriate antimicrobial therapy has an independent association with a lower 14-day mortality of the most severely ill patients.

Both appropriate and inappropriate antimicrobials prescribed to patients are analyzed (Tables 4 and 5 , respectively) and APACHE II scores among patient groups receiving different regimens were not significantly different. Among patients who received appropriate antimicrobial therapy, those receiving tigecycline-based or colistin-based therapy had a higher 14-day and 28-day mortality (Table 4), and no antimicrobial class was associated with a higher or lower 14-day and 28-day mortality after a multivariable analysis (data now shown).
Patients receiving tigecycline-based or colistin-based therapy had been infected carbapenem-resistant A. baumannii (CRAB) more frequently than those receiving other antimicrobial agents $(97.1 \%$ vs $57.3 \%, p<0.001)$. Among patients who received inappropriate antimicrobial therapy, patients receiving antipseudomonal penicillins had a higher 28-day mortality compared to other antimicrobial therapies after multivariable adjustment (data not shown). For patients infected with CRAB receiving appropriate antimicrobial therapy, carbapenem + tigecycline-based therapy was associated with a higher 14-day and 28-day mortality (univariate analysis, Supplemental Table S5), but no antimicrobial class was associated with a higher or lower 14- or 28-day mortality after multivariable adjustment. For patients infected with CRAB receiving inappropriate antimicrobial therapy, no antimicrobial class was associated with a higher or lower 14- or 28-day mortality (Supplemental Table S6). 
Table 5 Antimicrobial regimens for the treatment of Acinetobacter baumannii bacteremic pneumonia (inappropriate antibiotics)

\begin{tabular}{|c|c|c|c|c|c|c|c|}
\hline \multirow[t]{2}{*}{ Main agents used ${ }^{a, b}$} & \multirow{2}{*}{ 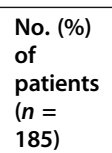 } & \multirow{2}{*}{$\begin{array}{l}\text { APACHE } \\
\text { II score, } \\
\text { median } \\
(\text { IQR) }\end{array}$} & \multicolumn{5}{|l|}{ No. (\%) of patients } \\
\hline & & & Combination therapy $^{e}$ & 14-Day Mortality & $P$-value & 28-Day Mortality & $P$-value \\
\hline Anti-pseudomonas penicillin-based therapy & $28(15.1)$ & $33(24-39)$ & $3(10.7)$ & $19(67.9)$ & .142 & $22(78.6)^{f}$ & .077 \\
\hline Anti-pseudomonas cephalosporin-based therapy & $36(19.5)$ & $31(21-40)$ & $8(22.2)$ & $17(47.2)$ & .287 & $22(61.1)$ & .710 \\
\hline Carbapenem-based therapy & $61(33.0)$ & $29(24-38)$ & $11(18.0)$ & $38(62.3)$ & .170 & $45(73.8)$ & .047 \\
\hline Colistin-based therapy & $7(3.8)$ & $28(21-30)$ & $7(100)$ & $3(42.9)$ & .702 & $6(85.7)$ & .425 \\
\hline Tigecycline-based therapy & $13(7.0)$ & $33(25-39)$ & $10(76.9)$ & $9(69.2)$ & .390 & $10(76.9)$ & .382 \\
\hline Fluoroquinolone-based therapy & $10(5.4)$ & $28(20-39)$ & $3(30.0)$ & $5(50.0)$ & .737 & $7(70.0)$ & 1 \\
\hline Sulbactam-based therapy & $10(5.4)$ & $30(18-37)$ & $6(60.0)$ & $5(50.0)$ & .737 & $7(70.0)$ & 1 \\
\hline Carbapenem + colistin-based therapy & $3(1.6)$ & $19,27,28$ & $1(33.3)$ & 0 & .088 & $3(100)$ & .555 \\
\hline Carbapenem + tigecycline-based therapy & $4(2.2)$ & $27(22-31)$ & $2(50.0)$ & $2(50.0)$ & 1 & $3(75.0)$ & 1 \\
\hline Carbapenem + sulbactam-based therapy & $5(2.7)$ & $31(29-31)$ & $3(60.0)$ & $3(60.0)$ & 1 & $4(80.0)$ & .655 \\
\hline Colistin + tigecycline-based therapy & $2(1.1)$ & 19,40 & $1(50.0)$ & $1(50.0)$ & 1 & $2(100)$ & .535 \\
\hline Carbapenem + colistin + tigecycline-based therapy & $1(0.5)$ & 19 & 0 & 0 & .449 & $1(100)$ & 1 \\
\hline \multicolumn{8}{|l|}{ Antimicrobial regimens $^{c}$} \\
\hline Anti-pseudomonas penicillin only & $25(13.5)$ & $33(24-39)$ & & $18(72.0)$ & .068 & $20(80.0)$ & .070 \\
\hline Anti-pseudomonas cephalosporin only & $28(15.1)$ & $32(22-41)$ & & $14(50.0)$ & .553 & $17(60.0)$ & .714 \\
\hline Carbapenem + colistin & $2(1.1)$ & 27,28 & & 0 & .200 & $2(100.0)$ & .535 \\
\hline Carbapenem + tigecycline & $2(1.1)$ & 25,33 & & $1(50.0)$ & 1 & $1(50.0)$ & 1 \\
\hline Carbapenem + sulbactam & $2(1.1)$ & 31,40 & & $2(100)$ & .503 & $2(100)$ & .535 \\
\hline Carbapenem + fluoroquinolone & $1(0.5)$ & 43 & & $1(100)$ & 1 & $1(100)$ & 1 \\
\hline Carbapenem + tigecycline + colistin & $1(0.5)$ & 19 & & 0 & .449 & $1(100)$ & 1 \\
\hline Tigecycline only & $3(1.6)$ & $30,33,38$ & & $3(100)$ & .254 & $3(100)$ & .555 \\
\hline Colistin + tigecycline & $1(0.5)$ & 40 & & $1(100)$ & 1 & $1(100)$ & 1 \\
\hline
\end{tabular}

${ }^{a}$ An antimicrobial agent (or antimicrobial agents)-based therapy denotes the corresponding antimicrobial agent(s) alone or in combination with other antimicrobial agent(s)

"Colistin" denotes intravenous colistin only. Inhaled colistin is not included

'Not in combination with other antimicrobial agents

${ }^{d} I Q R$, interquartile range. When the case number is less than 4, the APACHE II score for each case is shown

${ }^{\text {e}} \mathrm{Combination}$ therapy is defined as administration of more than one antimicrobial agent

f Patients receiving antipseudomonal penicillin therapy had a significantly higher 28-day mortality compared to other antimicrobial therapy after

multivariable adjustment

\section{Discussion}

The efficacy of antimicrobial therapy for A. baumannii bacteremic pneumonia has been difficult to establish due to the three phenotypically indistinguishable Acinetobacter species that make up the Ab group and the confounding influence of underlying diseases and severity of infection. This retrospective study analyzed the effect of appropriate antimicrobial therapy on the 14-day mortality of the patients with genomically identified monomicrobial A. baumannii bacteremic pneumonia after adjusting for multiple risk factors. We demonstrated that appropriate antimicrobial therapy lowers mortality in the most severely ill patients.

The impacts of appropriate antimicrobial therapy on patients might be modified by the illness severity. It has been found that inappropriate antimicrobial therapy seem to do less harm in non-severe cases and in the most severely ill patients with short life expectancies [26]. On the other hand, our previous study of
A. baumannii bacteremia patients showed that appropriate antimicrobial therapy reduced mortality in severely ill patients (APACHE II score $>25$ ) [18].. Another study on carbapenem nonsusceptible Klebsiella pneumoniae also suggested that appropriate antimicrobial therapy did not benefit non-severe patients (APACHE II < 15) [27]. Our observation that appropriate antimicrobial therapy may be of crucial importance to the survival of the most severely ill patients (APACHE II score $>35$ ) is in line with the previous studies. To our knowledge, this study is the first to explore the influence of severity of illness on the impacts of appropriate antimicrobial therapy in A. baumannii bacteremic pneumonia patients.

The pneumonia caused by phenotypically identified" $A$. baumannii" described in many studies actually comprises pneumonia caused by either one of the Acinetobacter species in the Ab group [13, 28]. There are differences in antimicrobial resistance and outcomes 
between A. baumannii and other Acinetobacter species in the $\mathrm{Ab}$ group [29-31], therefore pneumonia caused by these different Acinetobacter species cannot be considered as a single clinical entity. This study separated $A$. baumannii from other Acinetobacter species to avoid the confounding effect caused by the inclusion of a mixture of Acinetobacter species.

Colistin and tigecycline are often used for treatment of carbapenem-resistant Acinetobacter infections or as a salvage therapy for Acinetobacter infections with carbapenem treatment failure. However, our results showed that patients receiving tigecycline-based or colistin-based therapy, even both appropriate, still had a high 14- and 28-day mortality. Admittedly, patients receiving tigecycline-based or colistin-based therapy were more likely to have had been infected by CRAB strains and CRAB infection were associated with poorer outcome. However, tigecycline-based or colistin-based therapy was still not associated with a lower 14- and 28-day mortality for patients infected with CRAB. Colistin is administered as an inactive prodrug (colistin methanesulfonate) which results in a prolonged period of low plasma concentrations of the active drug and thereby influences its efficacy [32]. Possible explanations for tigecycline include its bacteriostatic property, a low AUC/MIC ratio [33-37], pneumonia as a source of bacteremia [36, 38], and relatively high MICs of tigecycline of our study isolates that were unachievable by the currently approved dose of tigecycline in the serum [21].

Among patients who received inappropriate antimicrobial therapy, patients receiving antipseudomonal penicillins had a higher 28-day mortality compared to other antimicrobial therapies. The similarity of APACHE II scores between patient groups receiving different regimens excludes disease severity as a confounder to explain the difference in mortality. Our finding suggested the potential detrimental effect of antipseudomonal penicillins for the treatment of $A$. baumannii bacteremic pneumonia. Further investigation is warranted to explore the cause of the finding.

Our study had some limitations. First, it is a retrospective study which is prone to selection bias and may limit the generalizability of our study. Second, our study included only patients with bacteremic pneumonia, thus the findings may not be applicable to A. baumannii pneumonia patients without bacteremia. The strengths of our study include the large case numbers obtained from multiple medical centers, genomically defined $A$. baumannii, and the adjustment of various risk factors.

\section{Conclusion}

Appropriate antimicrobial therapy decreases the 14-day mortality of the most severely ill patients with $A$. baumannii bacteremic pneumonia. Further research is needed to determine the most effective antimicrobial therapy for A. baumannii bacteremic pneumonia.

\section{Supplementary information}

Supplementary information accompanies this paper at https://doi.org/10. 1186/s13756-020-00824-4.

Additional file 1: Table S1. Adjusted odds ratios for appropriate antibiotics for 14-day mortality in patients with Acinetobacter baumannii bacteremic pneumonia: Stratified by APACHE II Score in quartiles. Table S2. Logistic regression of predictors for 14-day mortality in low APACHE score patients (APACHE Score $<36$ ) with Acinetobacter baumannii

bacteremic pneumonia. Table S3. Logistic regression of demographic characteristics and comorbidities for 14-day mortality in patients with Acinetobacter baumannii bacteremic pneumonia. Table S4. Odds ratios adjusting for gender and comorbidities for appropriate antibiotics for 14day mortality in patients with Acinetobacter baumannii bacteremic pneumonia: Stratified by APACHE II score categories. Table S5. Antimicrobial regimens for the treatment of carbapenem-resistant Acinetobacter baumannii bacteremic pneumonia (appropriate antibiotics). Table S6. Antimicrobial regimens for the treatment of carbapenem-resistant Acinetobacter baumannii bacteremic pneumonia (inappropriate antibiotics).

\section{Abbreviations}

A. baumannii: Acinetobacter baumannii; APACHE: Acute Physiology and Chronic Health Evaluation; AUC: Area under the curve; BAL: Bronchoalveolar lavage; Cl: Confidence interval; CRAB: Carbapenem-resistant Acinetobacter baumannii; CCH: Changhua Christian Hospital; ICU: Intensive care unit; MDR: Multiple drug resistant; MIC: Minimum inhibitory concentration; MMH: Mackay Memorial Hospital; NTUH: National Taiwan University Hospital; OR: Odds ratio; TSGH: Tri-Service General Hospital; TVGH: Taipei Veterans General Hospital; XDR: Extensively drug resistant

\section{Acknowledgements}

Not applicable.

\section{Authors' contributions}

F.Y.K, C.K.H, and Y.T.L. conceived the project and wrote the manuscript. F.Y.K, C.K.H, Y.T.L, and T.L.C designed the experiments and manuscript revisions. F.Y.K, Y.C.W, A. C, Y.S.Y, S.C.K, C.P.L, and Y.M.L. collected the clinical data. Y.C.W, and Y.T.L. performed experiments. F.Y.K, Y.T.L, and Y.C.W. conducted statistical analysis. All authors reviewed the manuscript. The authors read and approved the final manuscript.

\section{Funding}

This work was supported by grants from Taipei Veterans General Hospital (V106B-002, V107C-037, V108C-012, V109C-012, VTA106-T-5-3, VTA107-T-3-2, VTA108-T-2-2, VTA108-T-2-3 and VTA109-T-3-2), Tri-Service General Hospital (TSGH-C105-112, TSGH-105-113, TSGH-C106-096, TSGH-C107-099, TSGH-

C109-144, TSGH-E-109237, and VTA108-T-2-2), National Defense Medical Center (MAB-106-076 and MAB-106-098), and the Ministry of Science and Technology (MOST 104-2314-B-075-043-MY3, MOST 105-2314-B-016-039-MY3, MOST 105-2628-B-016-003-MY2, MOST 107-2314-B-075-066-MY3, MOST 1072314-B-016-051-MY3 and MOST 108-2314-B-016-029).

The funders had no role in the study design, data collection and interpretation, or the decision to submit the work for publication.

\section{Availability of data and materials}

All data generated or analyzed during this study are included in this published article and its supplementary information files. Original data are available from the corresponding author upon reasonable request.

\section{Ethics approval and consent to participate}

The study protocol was approved by the Research Ethics Committee of all participating hospitals. Informed consent was waived because of the retrospective nature of the study and the analysis used anonymous clinical data.

Competing interests

All authors declare that they have no competing interests. 


\section{Author details}

'Department of Emergency Medicine, Taipei Veterans General Hospital, No.201, Section 2, Shipai Road, Taipei 11217, Taiwan. ${ }^{2}$ Faculty of Medicine, School of Medicine, National Yang-Ming University, Taipei, Taiwan. ${ }^{3}$ Division of Infectious Diseases and Tropical Medicine, Department of Internal Medicine, Tri-Service General Hospital, National Defense Medical Center, Taipei, Taiwan. ${ }^{4}$ Department of Internal Medicine, National Taiwan University Hospital and National Taiwan University College of Medicine, Taipei, Taiwan. ${ }^{5}$ National Institute of Infectious Diseases and Vaccinology, National Health Research Institute, Miaoli County, Taiwan. ${ }^{6}$ Division of Infectious Diseases, Department of Internal Medicine, Mackay Memorial Hospital, Taipei, Taiwan. ${ }^{7}$ Department of Medical Research, Mackay Memorial Hospital, Taipei, Taiwan. ${ }^{8}$ Division of Infectious Diseases, Department of Internal Medicine, Changhua Christian Hospital, Changhua, Taiwan. ${ }^{9}$ Graduate Institute of Life Sciences, National Defense Medical Center, Taipei, Taiwan. ${ }^{10}$ Division of Infectious Diseases, Department of Medicine, Taipei Veterans General Hospital, Taipei, Taiwan.

Received: 2 June 2020 Accepted: 23 September 2020

Published online: 29 September 2020

\section{References}

1. Alp E, Güven M, Yildiz O, Aygen B, Voss A, Doganay M. Incidence, risk factors and mortality of nosocomial pneumonia in intensive care units: a prospective study. Ann Clin Microbiol Antimicrob. 2004;3:17.

2. Leu HSKD, Mori M, Woolson RF, Wenzel RP. Hospital-acquired pneumonia. Attributable mortality and morbidity. Am J Epidemiol. 1989;129(6):1258-67.

3. Chang HC, Chen YC, Lin MC, Liu SF, Chung YH, Su MC, et al. Mortality risk factors in patients with Acinetobacter baumannii ventilator: associated pneumonia. J Formos Med Assoc. 2011:110(9):564-71.

4. Cisneros-Herreros JM, Garnacho-Montero J, Pachon-lbanez ME. Nosocomia pneumonia due to Acinetobacter baumannii. Enferm Infecc Microbiol Clin. 2005;23(Suppl 3):46-51.

5. Fagon JY, Chastre J, Hance AJ, Montravers P, Novara A, Gibert C. Nosocomial pneumonia in ventilated patients: a cohort study evaluating attributable mortality and hospital stay. Am J Med. 1993;94(3):281-8.

6. Garnacho-Montero J, Ortiz-Leyba C, Fernandez-Hinojosa E, Aldabo-Pallas T, Cayuela A, Marquez-Vacaro JA, et al. Acinetobacter baumannii ventilatorassociated pneumonia: epidemiological and clinical findings. Intensive Care Med. 2005;31(5):649-55.

7. Koulenti D, Tsigou E, Rello J. Nosocomial pneumonia in 27 ICUs in Europe: perspectives from the EU-VAP/CAP study. Eur J Clin Microbiol Infect Dis. 2017;36(11):1999-2006

8. Brotfain E, Borer A, Koyfman L, Saidel-Odes L, Frenkel A, Gruenbaum SE, et al. Multidrug resistance Acinetobacter bacteremia secondary to ventilator-associated pneumonia: risk factors and outcome. J Intensive Care Med. 2017;32(9):528-34.

9. Ellis D, Cohen B, Liu J, Larson E. Risk factors for hospital-acquired antimicrobial-resistant infection caused by Acinetobacter baumannii. Antimicrob Resist Infect Control. 2015;4:40

10. Kanafani ZA, Zahreddine N, Tayyar R, Sfeir J, Araj GF, Matar GM, et al. Multidrug resistant Acinetobacter species: a seven-year experience from a tertiary care center in Lebanon. Antimicrob Resist Infect Control. 2018;7:9.

11. Čiginskienė A, Dambrauskienė A, Rello J, Adukauskienė D. Ventilatorassociated pneumonia due to drug-resistant Acinetobacter baumannii: risk factors and mortality relation with resistance profiles, and independent predictors of in-hospital mortality. Medicina (Kaunas). 2019;55(2):49.

12. Sunenshine RH, Wright MO, Maragakis LL, Harris AD, Song X, Hebden J, et al. Multidrug-resistant Acinetobacter infection mortality rate and length of hospitalization. Emerg Infect Dis. 2007;13(1):97-103.

13. Joung MK, Kwon KT, Kang Cl, Cheong HS, Rhee JY, Jung DS, et al. Impact of inappropriate antimicrobial therapy on outcome in patients with hospital-acquired pneumonia caused by Acinetobacter baumannii. J Inf Secur. 2010;61(3):212-8.

14. Zheng YL, Wan YF, Zhou LY, Ye ML, Liu S, Xu CQ, et al. Risk factors and mortality of patients with nosocomial carbapenem-resistant Acinetobacter baumannii pneumonia. Am J Infect Control. 2013;41(7):e59-63.

15. Chen HP, Chen TL, Lai CH, Fung CP, Wong WW, Yu KW, et al. Predictors of mortality in Acinetobacter baumannii bacteremia. J Microbiol Immunol Infect. 2005;38(2):127-36.

16. Chusri S, Chongsuvivatwong V, Silpapojakul K, Singkhamanan K, Hortiwakul T, Charernmak B, Doi Y. Clinical characteristics and outcomes of community and hospital-acquired Acinetobacter baumannii bacteremia. J Microbiol Immunol Infect. 2019;52(5):796-806.

17. Erbay A, Idil A, Gozel MG, Mumcuoglu I, Balaban N. Impact of early appropriate antimicrobial therapy on survival in Acinetobacter baumannii bloodstream infections. Int J Antimicrob Agents. 2009;34(6):575-9.

18. Lee YT, Kuo SC, Yang SP, Lin YT, Tseng FC, Chen TL, et al. Impact of appropriate antimicrobial therapy on mortality associated with Acinetobacter baumannii bacteremia: relation to severity of infection. Clin Infect Dis. 2012; 55(2):209-15

19. Garonzik SM, Li J, Thamlikitkul V, Paterson DL, Shoham S, Jacob J, et al. Population pharmacokinetics of colistin methanesulfonate and formed colistin in critically ill patients from a multicenter study provide dosing suggestions for various categories of patients. Antimicrob Agents Chemother. 2011;55(7):3284-94.

20. Pogue JM, Ortwine JK, Kaye KS. Clinical considerations for optimal use of the polymyxins: a focus on agent selection and dosing. Clin Microbiol Infect. 2017;23(4):229-33.

21. Chen TL, Siu LK, Wu RC, Shaio MF, Huang LY, Fung CP, et al. Comparison of one-tube multiplex PCR, automated ribotyping and intergenic spacer (ITS) sequencing for rapid identification of Acinetobacter baumannii. Clin Microbiol Infect. 2007;13(8):801-6.

22. Poirel L, Jayol A, Nordmann P. Polymyxins: antibacterial activity, susceptibility testing, and resistance mechanisms encoded by plasmids or chromosomes. Clin Microbiol Rev. 2017;30(2):557-96.

23. Hindler JA, Humphries RM. Colistin MIC variability by method for contemporary clinical isolates of multidrug-resistant gram-negative bacilli. J Clin Microbiol. 2013:51(6):1678-84.

24. Bradford PA, Petersen PJ, Young M, Jones $\mathrm{CH}$, Tischler M, O'Connell J. Tigecycline MIC testing by broth dilution requires use of fresh medium or addition of the biocatalytic oxygen-reducing reagent oxyrase to standardize the test method. Antimicrob Agents Chemother. 2005;49(9):3903-9.

25. Hosseini M, Ramazani J. Evaluation of acute physiology and chronic health evaluation II and sequential organ failure assessment scoring systems for prognostication of outcomes among intensive care Unit's patients. Saudi J Anaesth. 2016;10(2):168-73.

26. Harbarth S, Nobre V, Pittet D. Does antibiotic selection impact patient outcome? Clin Infect Dis. 2007;44(1):87-93.

27. Lin YT, Chuang C, Su CF, Chan YJ, Wang LS, Huang CT, et al. Efficacy of appropriate antimicrobial therapy on the survival of patients with Carbapenem nonsusceptible Klebsiella Pneumoniae infection: a multicenter study in Taiwan. Medicine (Baltimore). 2015;94(33):e1405.

28. Garnacho J, Sole-Violan J, Sa-Borges M, Diaz E, Rello J. Clinical impact of pneumonia caused by Acinetobacter baumannii in intubated patients: a matched cohort study. Crit Care Med. 2003;31(10):2478-82.

29. Chuang YC, Sheng WH, Li SY, Lin YC, Wang JT, Chen YC, et al. Influence of genospecies of Acinetobacter baumannii complex on clinical outcomes of patients with acinetobacter bacteremia. Clin Infect Dis. 2011;52(3):352-60.

30. Lee NY, Chang TC, Wu CJ, Chang CM, Lee HC, Chen PL, et al. Clinical manifestations, antimicrobial therapy, and prognostic factors of monomicrobial Acinetobacter baumannii complex bacteremia. J Inf Secur. 2010;61 (3):219-27.

31. Wisplinghoff $H$, Paulus $T$, Lugenheim M, Stefanik D, Higgins PG, Edmond $\mathrm{MB}$, et al. Nosocomial bloodstream infections due to Acinetobacter baumannii, Acinetobacter pittii and Acinetobacter nosocomialis in the United States. J Infect. 2012;64(3):282-90.

32. Tran $T B$, Velkov $T$, Nation $R L$, Forrest $A$, Tsuji BT, Bergen $P J$, et al. Pharmacokinetics/pharmacodynamics of colistin and polymyxin B: are we there yet? Int J Antimicrob Agents. 2016;48(6):592-7.

33. Cheng A, Chuang YC, Sun HY, Sheng WH, Yang CJ, Liao CH, et al. Excess mortality associated with Colistin-Tigecycline compared with ColistinCarbapenem combination therapy for extensively drug-resistant Acinetobacter baumannii bacteremia: a multicenter prospective observational study. Crit Care Med. 2015;43(6):1194-204.

34. Bhavnani SM, Rubino CM, Hammel JP, Forrest A, Dartois N, Cooper CA, et al. Pharmacological and patient-specific response determinants in patients with hospital-acquired pneumonia treated with tigecycline. Antimicrob Agents Chemother. 2012;56(2):1065-72.

35. Ambrose PG, Hammel JP, Bhavnani SM, Rubino CM, Ellis-Grosse EJ, Drusano GL. Frequentist and Bayesian pharmacometric-based approaches to facilitate critically needed new antibiotic development: overcoming lies, damn lies, and statistics. Antimicrob Agents Chemother. 2012;56(3):1466-70. 
36. Liou BH, Lee YT, Kuo SC, Liu PY, Fung CP. Efficacy of tigecycline for secondary Acinetobacter bacteremia and factors associated with treatment failure. Antimicrob Agents Chemother. 2015;59(6):3637-40.

37. Stein GE, Babinchak T. Tigecycline: an update. Diagn Microbiol Infect Dis. 2013;75(4):331-6.

38. Chuang YC, Cheng CY, Sheng WH, Sun HY, Wang JT, Chen YC, et al. Effectiveness of tigecycline-based versus colistin- based therapy for treatment of pneumonia caused by multidrug-resistant Acinetobacter baumannii in a critical setting: a matched cohort analysis. BMC Infect Dis. 2014;14:102.

\section{Publisher's Note}

Springer Nature remains neutral with regard to jurisdictional claims in published maps and institutional affiliations.

Ready to submit your research? Choose BMC and benefit from:

- fast, convenient online submission

- thorough peer review by experienced researchers in your field

- rapid publication on acceptance

- support for research data, including large and complex data types

- gold Open Access which fosters wider collaboration and increased citations

- maximum visibility for your research: over $100 \mathrm{M}$ website views per year

At $B M C$, research is always in progress.

Learn more biomedcentral.com/submissions 